\title{
Pediatric Hospital Discharge Interventions to Reduce Subsequent Utilization: A Systematic Review
}

\author{
Katherine A. Auger, MD, MSc ${ }^{1 \star}$, Chén C. Kenyon, MD, MSHP², Chris Feudtner, MD, PhD, MPH ${ }^{3}$, Matthew M. Davis, MD, MAPP4
}

${ }^{1}$ Department of Pediatrics, Division of Hospital Medicine, James M. Anderson Center for Health Care Excellence, Cincinnati Children's Hospital Medical Center, Cincinnati, Ohio; '2Department of Pediatrics, Division of General Pediatrics, The Children's Hospital of Philadelphia, Philadelphia, Pennsylvania; ${ }^{3}$ Department of Pediatrics, Division of General Pediatrics, Department of Medical Ethics. The Children's Hospital of Philadelphia, Philadelphia, Pennsylvania; 'Department of Pediatrics and Communicable Diseases, Division of General Pediatrics, Department of Internal Medicine, Institute for Healthcare Policy and Innovation, Gerald R. Ford School of Public Policy, University of Michigan, Ann Arbor, Michigan.

BACKGROUND: Reducing avoidable readmission and posthospitalization emergency department (ED) utilization has become a focus of quality-of-care measures and initiatives. For pediatric patients, no systematic efforts have assessed the evidence for interventions to reduce these events.

PURPOSE: We sought to synthesize existing evidence on pediatric discharge practices and interventions to reduce hospital readmission and posthospitalization ED utilization.

DATA SOURCES: PubMed and the Cumulative Index to Nursing and Allied Health Literature.

STUDY SELECTION: Studies available in English involving pediatric inpatient discharge interventions with at least 1 outcome of interest were included.

DATA EXTRACTION: We utilized a modified Cochrane Good Practice data extraction tool and assessed study quality with the Downs and Black tool.

DATA SYNTHESIS: Our search identified a total of 1296 studies, 14 of which met full inclusion criteria. All included studies examined multifaceted discharge interventions initiated in the inpatient setting. Overall, 2 studies demonstrated statistically significant reductions in both readmissions and subsequent ED visits, 4 studies demonstrated statistically significant reductions in either readmissions or ED visits, and 2 studies found statistically significant increases in subsequent utilization. Several studies were not sufficiently powered to detect changes in either subsequent utilization outcome measure.

CONCLUSIONS: Interventions that demonstrated reductions in subsequent utilization targeted children with specific chronic conditions, providing enhanced inpatient feedback and education reinforced with postdischarge support. Interventions seeking to reduce subsequent utilization should identify an individual or team to assume responsibility for the inpatient-to-outpatient transition and offer ongoing support to the family via telephone or home visitation following discharge. Journal of Hospital Medicine 2014;9:251-260. (c) 2013 Society of Hospital Medicine
The process of discharging a pediatric patient from an acute care facility is currently fraught with difficulties. More than $20 \%$ of parents report problems in the transition of care from the hospital to the home and ambulatory care setting. ${ }^{1}$ Clinical providers likewise note communication challenges around the time of discharge, ${ }^{2,3}$ especially when inpatient and outpatient providers are different, as with the hospitalist model. ${ }^{4}$ Poor communication and problems in discharge transition and continuity of care often culminate in adverse events, ${ }^{5,6}$ including return to emergency department (ED) care and hospital readmission. ${ }^{7}$

Thirty-day readmissions are common for certain pediatric conditions, such as oncologic diseases, transplantation, and sickle cell anemia and vary signifi-

\footnotetext{
*Address for correspondence and reprint requests: Katherine Auger, MD, 3333 Burnet Ave., MLC 9016; Cincinnati, OH 45229; Telephone: 513-636-3753; Fax: 513-636-4402; E-mail: katherine.auger@cchmc.org

Additional Supporting Information may be found in the online version of this article.

Received: October 1, 2013; Revised: November 15, 2013; Accepted: November 23, 2013

2013 Society of Hospital Medicine DOI 10.1002/jhm.2134

Published online in Wiley Online Library (Wileyonlinelibrary.com).
}

cantly across children's hospitals. ${ }^{8}$ Discharge planning may decrease 30-day readmissions in hospitalized adults $^{9}$; however, it is not clear that the same is true in children. Both the preventability of pediatric readmissions $^{10}$ and the extent to which readmissions reflect suboptimal care ${ }^{11}$ are subjects of debate. Despite these uncertainties, collaborative efforts intended to decrease pediatric readmissions ${ }^{12}$ and improve discharge transitions ${ }^{13,14}$ are underway.

To inform these debates and efforts, we undertook a systematic review of the evidence of hospitalinitiated interventions to reduce repeat utilization of the ED and hospital. Acknowledging that existing evidence for condition-specific discharge interventions in pediatrics might be limited, we sought to identify common elements of successful interventions across pediatric conditions.

\section{METHODS}

\section{Search Strategy}

With the assistance of a research librarian, we searched MEDLINE and CINAHL (Cumulative Index to Nursing and Allied Health Literature) from the inception of these databases through to March 28, 2012 (for search strategies, see the Supporting 
Information, Appendix, Part 1, in the online version of this article).

\section{Study Selection}

Two authors (K.A. and C.K.) independently reviewed abstracts identified by the initial search, as well as abstracts of references of included articles. Eligibility criteria for inclusion in full review included: (1) discharge-oriented process or intervention initiated in the inpatient setting, (2) study outcomes related to subsequent utilization including hospital readmission or emergency department visit after hospitalization, (3) child- or adolescent-focused or child-specific results presented separately, and (4) written or available in English. If abstract review did not sufficiently clarify whether all eligibility criteria were met, the article was included in the full review. Two authors (K.A. and C.K.) independently reviewed articles meeting criteria for full review to determine eligibility. Disagreements regarding inclusion in the final analysis were discussed with all 4 authors. We excluded studies in countries with low or lower-middle incomes, ${ }^{15}$ as discharge interventions in these countries may not be broadly applicable.

\section{Data Abstraction, Quality Assessment, and Data Synthesis}

Two authors (K.A. and C.K.) independently abstracted data using a modified Cochrane Collaboration data collection form. ${ }^{16}$ We independently scored the included studies using the Downs and Black checklist, which assesses the risk of bias and the quality of both randomized and nonrandomized studies. ${ }^{17}$ This checklist yields a composite score of 0 to 28 points, excluding the item assessing power. As many studies either lacked power calculations or included power calculations based on outcomes not included in our review, we performed calculations to determine the sample size needed to detect a decrease in readmission or ED utilization by $20 \%$ from baseline or control rates. Due to the heterogeneous nature of included studies in terms of population, interventions, study design, and outcomes, meta-analysis was not performed.

\section{RESULTS}

Electronic search yielded a total of 1296 unique citations. Review of abstracts identified 40 studies for full article review. We identified 10 articles that met all inclusion criteria. Subsequent review of references of included articles identified 20 additional articles for full review, 7 of which met all inclusion criteria. However, 3 articles $^{18-20}$ assessed the impact of violence interventions primarily on preventing reinjury and recidivism and thus were excluded (see Supporting Information, Appendix, Part 2, in the online version of this article for findings of the 3 articles). In total, we included 14 articles in our review ${ }^{21-34}$ (Figure 1).

\section{Patient Populations and Intervention Timing and Components}

Studies varied regarding the specific medical conditions they evaluated. Eight of the papers reported discharge interventions for children with asthma, 5 papers focused on discharge from the neonatal intensive care unit (NICU), and a final study discussed a discharge intervention for children with cancer (Table 1). Although our primary goal was to synthesize discharge interventions across pediatric conditions, we provide a summary of discharge interventions by condition (see Supporting Information, Appendix, Part 3, in the online version of this article).

Studies varied regarding the timing and nature of the intervention components. Eight discharge interventions included a major inpatient component, in addition to outpatient support or follow-up. 21,23-26,29,32,34 Two studies included an inpatient education component only. ${ }^{22,27}$ The remainder were initiated during index hospitalization but focused primarily on home visitation, enhanced follow-up, and support after discharge (Figure 2). ${ }^{28,30,31,33}$

\section{Outcome Assessment Methods}

Readmission and subsequent ED utilization events were identified using multiple techniques. Some authors accessed claims records to capture all outcomes. ${ }^{30,33}$ Others relied on chart review. ${ }^{21,25-28,31,32}$ One study supplemented hospital records with outpatient records. $^{24}$ Some investigators used parental reports. $^{22,23,31}$ Two studies did not describe methods for identifying postdischarge events. ${ }^{29,34}$

\section{Study Quality}

The quality of the included studies varied (Table 2). Many of the studies had inadequate sample size to detect a difference in either readmission or ED visit subsequent to discharge. Eight studies found differences in either subsequent ED utilization, hospitalization, or both and were considered adequately powered for these specific outcomes. ${ }^{21,23,25,26,28,30-32}$ In contrast, among studies with readmission as an outcome, 6 were not adequately powered to detect a difference in this particular outcome. ${ }^{24,30-34}$ In these 6 studies, all except 1 study $^{30}$ had $<10 \%$ of the sample size required to detect differences in readmission. Further, 2 studies that examined ED utilization were underpowered to detect differences between intervention and control groups. ${ }^{24,26}$ We were unable to perform power calculations for 3 studies, ${ }^{22,27,29}$ as the authors presented the number of events without clear denominators.

Excluding the assessment of statistical power, Downs and Black scores ranged from 10 to 23 (maximum 28 possible points) indicating varying quality. 


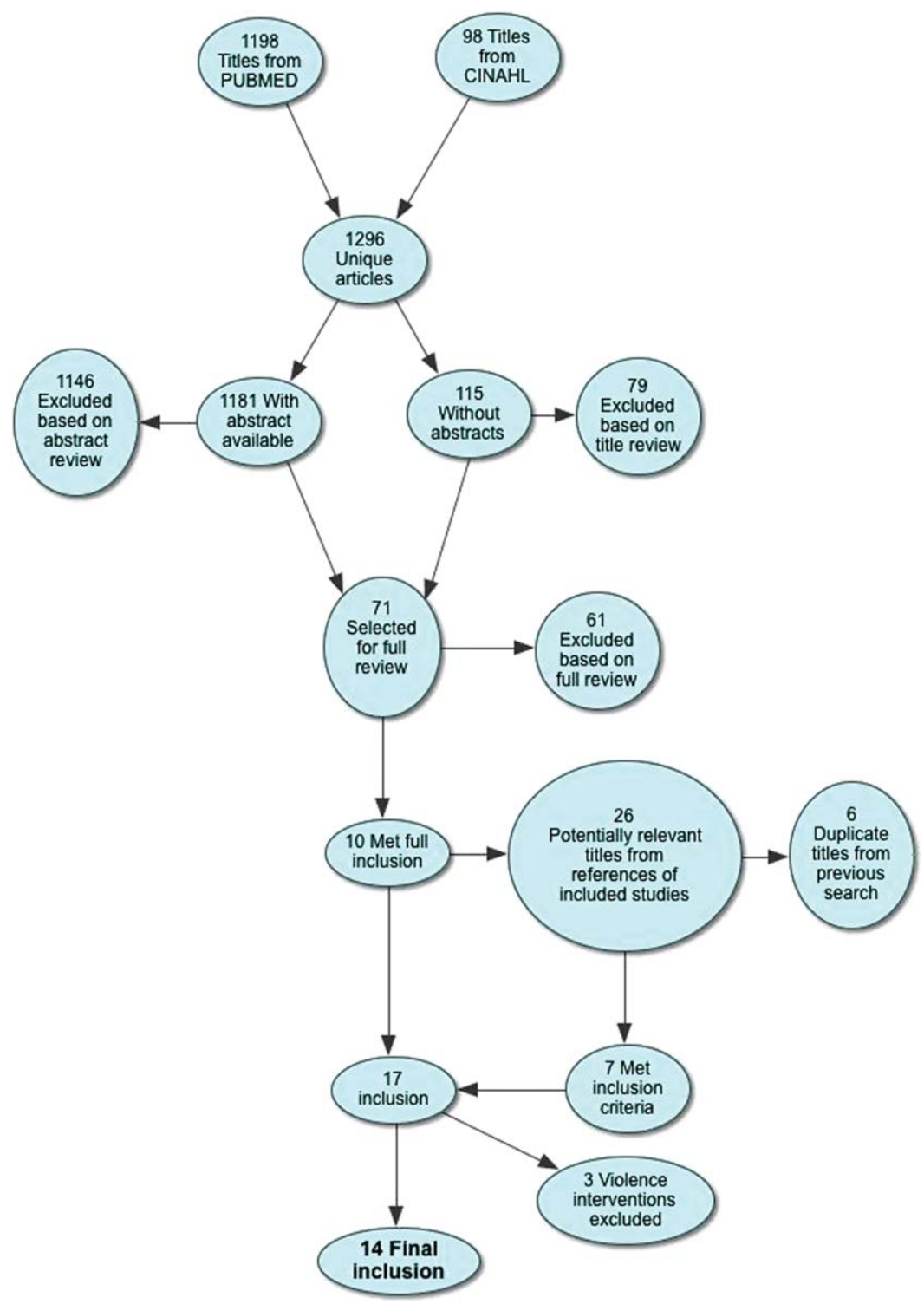

FIG. 1. Study inclusion. CINAHL, Cumulative Index to Nursing and Allied Health Literature.

As would be expected with discharge interventions, studies did not blind participants; 2 studies did, however, appropriately blind the outcome evaluators to intervention assignment. ${ }^{22,30}$ Even though 10 out of the 14 studies were randomized controlled trials, randomization may not have been completely effective due to sample size being too small for effective randomization, ${ }^{31}$ large numbers of excluded subjects after randomization, ${ }^{30}$ and unclear randomization process. ${ }^{34}$ Several studies had varying follow-up periods for patients within a given study. For example, 3 NICU studies assessed readmission at 1-year corrected age, ${ }^{30,31,33}$ creating the analytic difficulty that the amount of time a given patient was at risk for readmission was dependent on when the patient was discharged, yet this was not accounted for in the analyses. Only 2 studies demonstrated low rates of loss to follow-up $(<10 \%) .^{30,33}$ The remainder of the studies either had high incompletion/loss to follow-up rates $(>10 \%)^{22,24,31}$ or did not report rates..$^{21,23,25-}$ 29,32,34 Finally, 3 studies recruited patients from multiple sites, ${ }^{24,31,33}$ and none adjusted for potential differences in effect based on enrollment site.

\section{Findings Across Patient Populations Regarding Readmission}

Of the 4 studies that demonstrated change in overall readmission, ${ }^{23,25,26,28}$ all were asthma focused; $3 \mathrm{dem}$ onstrated a decrease in readmissions, ${ }^{23,25,26}$ and 1 an increase in readmissions. ${ }^{28}$ The 3 effective interventions included 1-on-1 inpatient education delivered by an asthma nurse, in addition to postdischarge followup support, either by telephone or clinic visit. Two of these interventions provided rescue oral steroids to some patients on discharge. ${ }^{25,26}$ In contrast, a study from New Zealand evaluated a series of postdischarge 


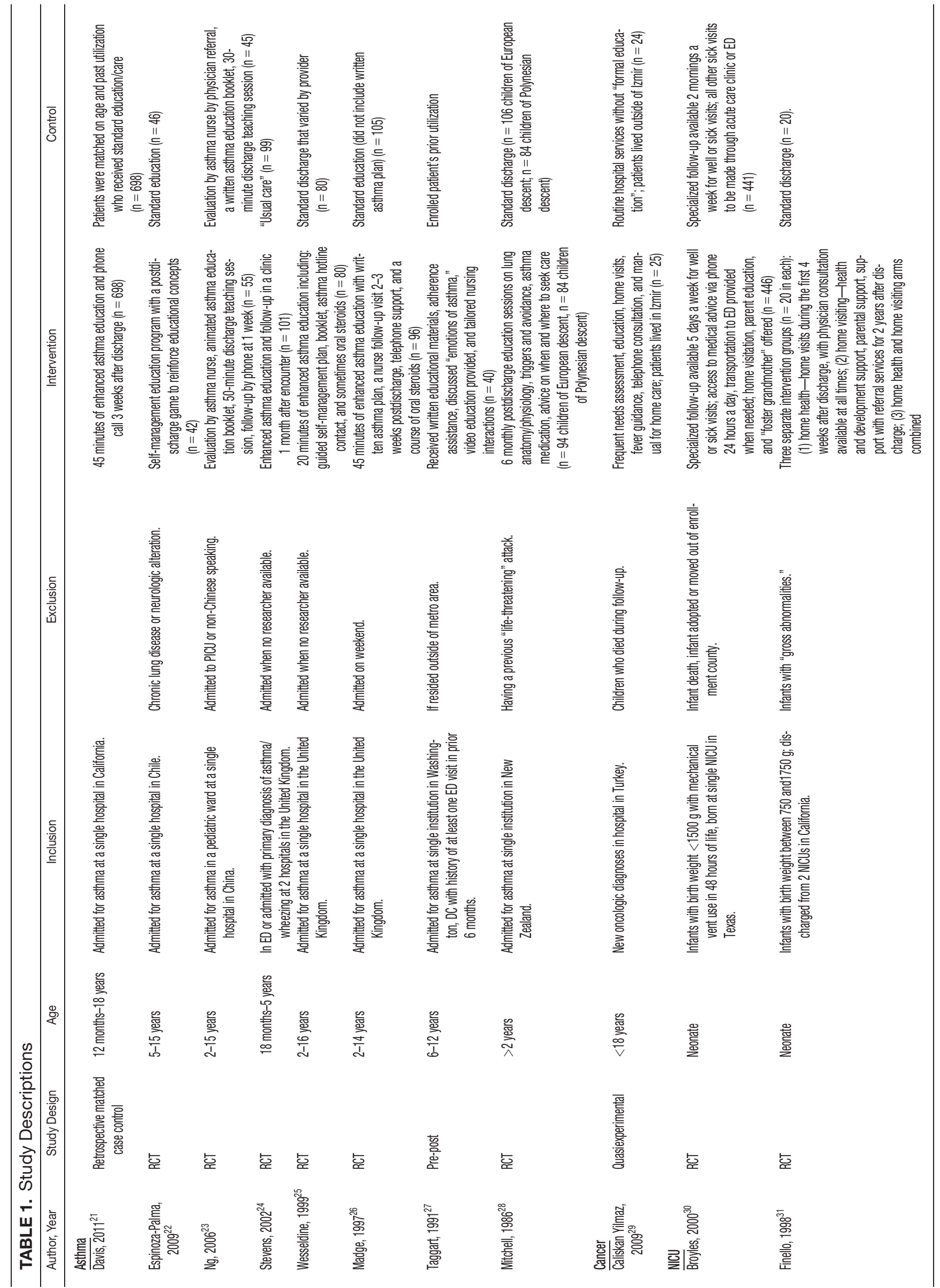


visits using an existing public health nurse infrastructure and demonstrated an increase in readmission between 6 to 18 months after admission in European children. ${ }^{28}$ An additional study focused on outpatient support after discharge from the NICU, and demonstrated a lower frequency of readmission to the intensive care unit without overall reduction of hospital readmission (Tables 1 and 2). ${ }^{30}$

\section{Findings Across Patient Populations Regarding Subsequent ED Visits}

Of all the discharge interventions, 6 demonstrated differences in return to the ED after discharge. Five studies described a decrease in ED visits after hospitalization, ${ }^{23,25,30-32}$ and 1 showed an increase. ${ }^{21}$ Three studies in the NICU population demonstrated decreased ED utilization through a combination of augmented family engagement during hospitalization and/or enhanced support after discharge. Two inpatient asthma education interventions with structured postdischarge follow-up decreased return visitation to the ED. ${ }^{23,26}$ The intervention that worsened subsequent ED utilization (ie, increased ED visit hazard compared to matched controls) provided enhanced inpatient education to a nonrandom group of children hospitalized with asthma and provided a follow-up phone call 3 weeks after discharge (Tables 1 and 2). ${ }^{21}$

\section{DISCUSSION}

In this review, we synthesized evidence regarding pediatric hospital discharge-focused interventions intended to reduce subsequent utilization through decreased readmission and ED visits. Our review identified 14 studies clustered in 3 clinical areas: asthma, NICU care (chiefly prematurity), and cancer. Overall, 6 interventions demonstrated a reduction either in subsequent hospitalization or ED use. Four of the 6 positive interventions included both an enhanced inpatient education and engagement component as well as enhanced follow-up after discharge. Importantly, all of the interventions were multifaceted; thus, we could not ascertain which specific aspects of the interventions mediated the change. Many of the included studies had significant methodological limitations.

\section{Current Conceptual Framework}

There are a number of existing discharge transitional care frameworks from prior studies ${ }^{35,36}$ and professional societies. ${ }^{37}$ The Stepping Up to the Plate (SUTTP) alliance, a collaborative of 9 professional organizations, including the American Academy of Pediatrics, introduced 1 such framework in 2007. SUTTP sought to enhance care transitions by outlining principles of discharge transitional care including: (1) enhanced accountability, (2) creation of a central "coordination hub" charged with communicating expectations for care, (3) clear and direct communication of treatment plans and follow-up, (4) timely feedback/feed-forward 


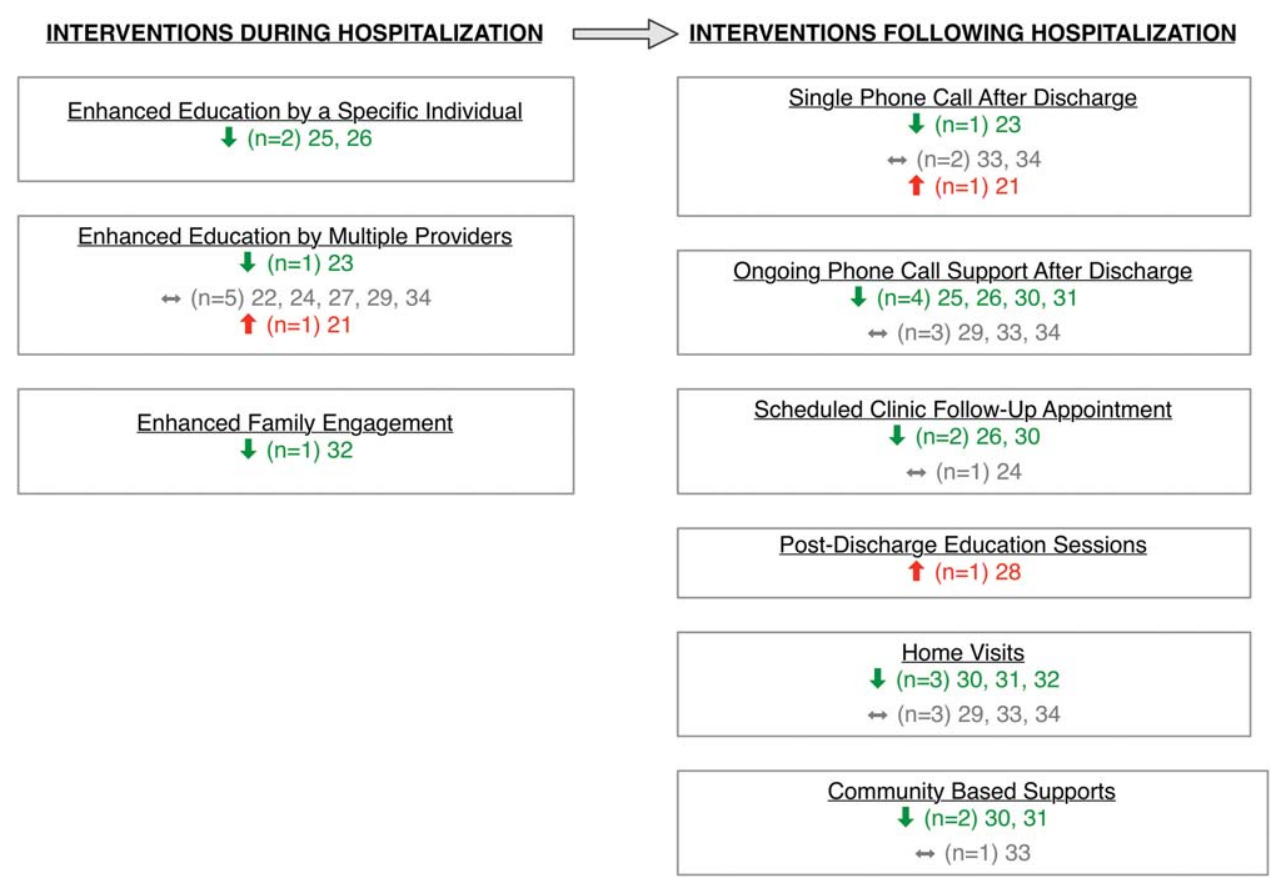

FIG. 2. Studies in green indicate improved/decreased subsequent utilization. Studies in gray indicate no change. Studies in red indicate worsened/increased subsequent utilization.

of relevant information, and (5) involvement of family member at every stage. ${ }^{38}$ In the context of the SUTTP framework, we present 3 hypotheses based on our findings to guide future work.

\section{Hypothesis: Appointing a Dedicated Individual or Coordinat- ing Hub Reduces Subsequent Utilization}

Ostensibly, each discharge intervention included in this review sought to enhance accountability of providers or their health systems for discharge transitional care. Two of the asthma interventions appointed a particular provider to coordinate the discharge transition and demonstrated reductions in readmission. ${ }^{25,26}$ The successful NICU discharge interventions provided an integrated accountability structure across the health system, with a transition of accountability to an outpatient provider or central coordinating hub available to provide assistance and resources for an extended period following discharge.

By contrast, interventions with more than 1 individual intervener or without a centrally coordinated system for discharge transitional care tended not to demonstrate reduction in subsequent utilization. $^{21,24,27,28}$ In fact, the 1 asthma intervention that utilized a previously existing public health nurse infrastructure demonstrated an increase in readmission. ${ }^{28}$ Future efforts to enhance transitional care might investigate directly the impact of accountability structure on subsequent utilization by varying the number of effector individuals or the organization to which they report (eg, hospital system vs public health department).

\section{Hypothesis: Individualized Task Learning and Feedback Enhances Effectiveness}

Studies varied with respect to the extent they incorporated the principles of enhanced communication of the treatment and follow-up plan and timely feedback/feed-forward of relevant information. Successful efforts, however, seemed to embrace these strategies. Each of the 3 interventions that demonstrated readmission reduction ${ }^{23,25,26}$ developed an individualized treatment plan during hospitalization, with either a specific follow-up plan or resources for outpatient support. Two of these interventions assessed asthma inhaler technique prior to discharge, creating an inpatient audit and feedback loop allowing for assessment of competence prior to discharge. Audit and feedback has demonstrated promise modifying provider behavior $^{39}$ and is a plausible approach to enhancing patient and family self-care.

\section{Hypothesis: Timing of Intervention Enhances Effectiveness}

Discrete sentinel events such as inpatient admission, may serve as a "teachable moment" 40,41 or a "tipping point" 42 for some patients/families to initiate behavior change. Four of the 6 positive studies had a robust inpatient education component. By providing enhanced inpatient support, providers may be engaging the family at a timely opportunity to improve care. Both timing of the intervention (at admission vs discharge) and content (education- vs familyengagement focused) are likely important to their effect and should be further explored with prospective study. 


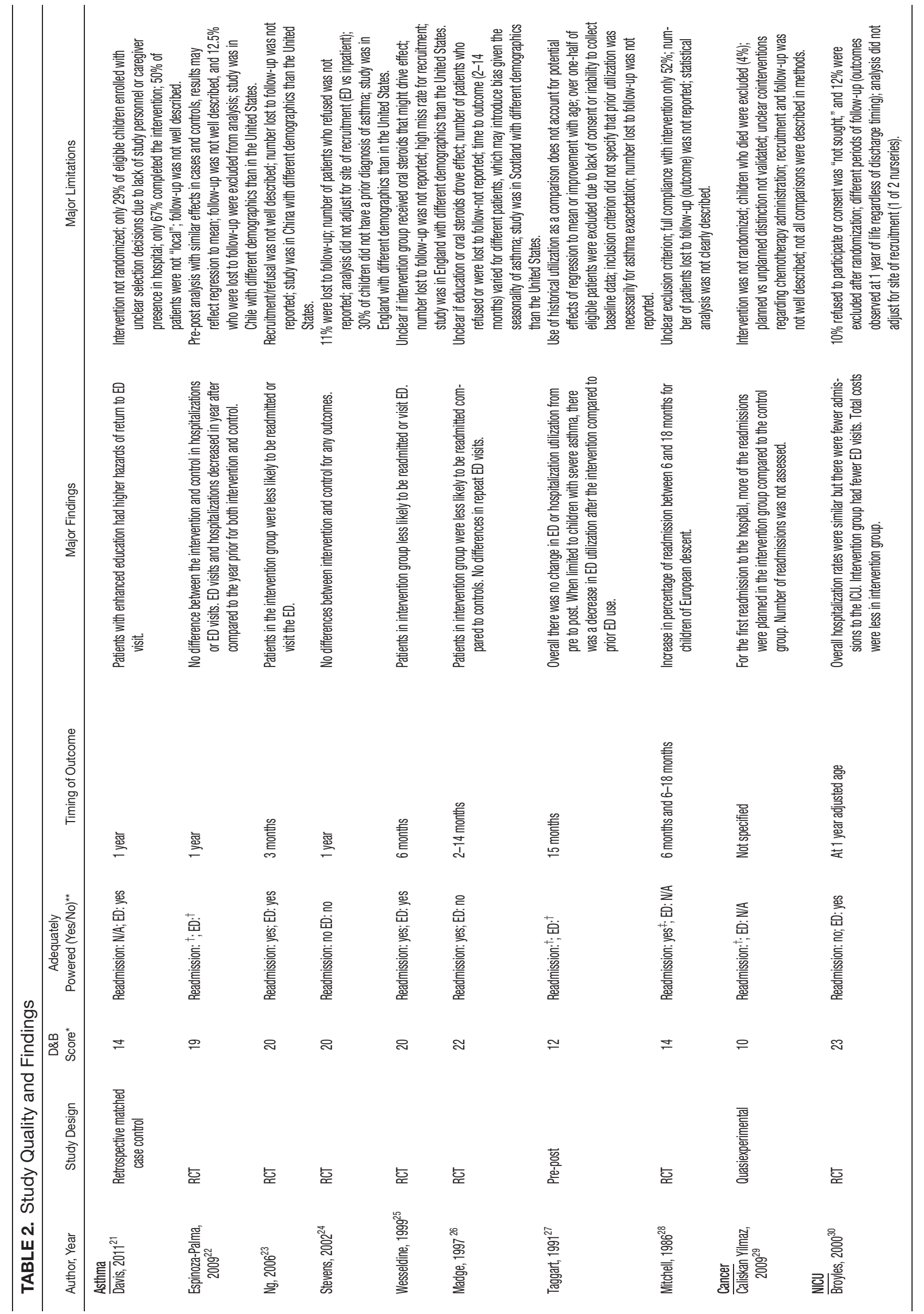




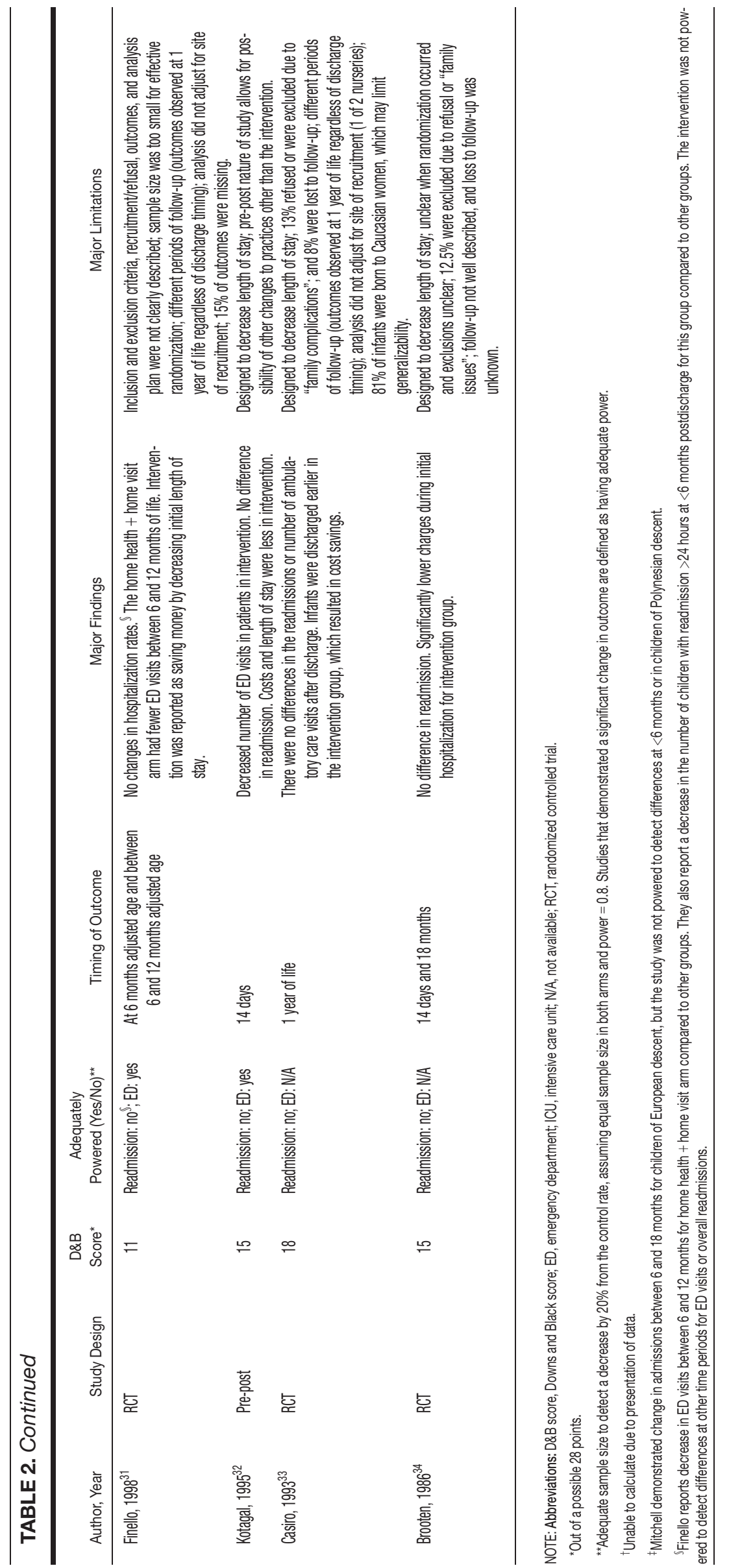




\section{Persistent Literature Gaps}

Follow-up with a primary care provider after discharge is another intervention that might decrease postdischarge utilization. We did not identify any studies that specifically examined primary care followup. However, 2 studies ${ }^{43,44}$ that did not meet our inclusion criteria (because they included adults and did not stratify by age group in the analysis) examined any outpatient follow-up after discharge using statespecific Medicaid claims. One study found that outpatient follow-up after sickle cell hospitalization was associated with lower rates of readmission. ${ }^{43}$ The other found no difference in readmission across multiple conditions. ${ }^{44}$ One recent review of outpatient follow-up from the ED for asthma found that even when increases in follow-up were achieved, no reduction in the subsequent utilization was observed. ${ }^{45}$

Additional important questions remain underexplored. First, are condition-specific interventions superior to those that span conditions? All of the interventions that demonstrated reductions in readmission were condition-specific, yet no generic interventions met our inclusion criteria. Importantly, only 1 study $^{29}$ in our review examined discharge processes from 1 of the pediatric conditions with the most variation $^{8}$ in readmission. Further, no studies focused on children with complex medical conditions, who are known to be at increased risk of readmission, ${ }^{46}$ indicating a sizable knowledge gap persists in understanding how to prevent readmissions in the most vulnerable pediatric populations.

Lastly, who are the most appropriate effector individuals for discharge-focused transitional care interventions? Demographically matched effector individuals have shown promise in improving care using community health workers. ${ }^{47,48}$ The degree to which the identity of the intervener mediates subsequent ED and hospital utilization warrants further investigation.

\section{Limitations of This Systematic Review}

The studies included in this review assessed different outcomes at different intervals, precluding metaanalysis. With greater consistency in the collection of data on the quality of discharge processes and their subsequent outcomes, future studies may offer further clarity as to which discharge-oriented practices are more effective than others. Because we only identified literature in 3 pediatric conditions, generalizability beyond these conditions may be limited. The settings of the interventions also occurred in multiple countries; we excluded countries from low or low-middle incomes to facilitate generalizability. As many of the discharge processes contained multiple interventions, it is not possible to ascertain which, if any, singular action may decrease posthospitalization utilization. Additionally, some of the included interventions are older, and it is plausible that discharge processes have evolved with the expansion of the hospitalist model.
Methods of data collection influence the quality of results in the included studies. Most of the studies included in this review used either medical record review or parental self-report of utilization. Parental report may be sufficient for hospitalizations and ED utilization; however, it is subject to recall bias. Chart review likely underestimates the number of postdischarge events, depending on the individual institution's proportion of the market and the tendency of individuals to seek care at multiple institutions. Claims data may offer the most accurate assessments of ED and hospital utilization and cost, but can be more difficult to obtain and do not provide the same potential for granularity as parent report or medical records review.

Finally, subsequent ED visits, readmissions, and cost may not be the best measures of the quality of discharge transitional care. A number of tools have been developed to more specifically evaluate the quality of transitional care in adults, ${ }^{49,50}$ including a validated instrument that consists of only 3 items, ${ }^{50}$ which primarily assesses the extent to which patients are prepared for self-care upon discharge. For pediatric populations, validated tools assessing caregiver experience with discharge ${ }^{51}$ and discharge readiness ${ }^{52}$ are also available. These instruments may assist those interested in assessing process-related outcomes that specifically assess discharge transitional care elements and may mediate subsequent ED visits or hospitalizations.

\section{CONCLUSION}

Successful discharge interventions to reduce pediatric readmission and $\mathrm{ED}$ have some common features, including an individual or team with specialized knowledge of the condition that assumed responsibility for the inpatient-to-outpatient transition and offered ongoing support to the family following discharge. All studies included in our review examined multiple discharge interventions; however, many did not have enough participants to detect differences in the outcomes of interest. Future studies might adapt common features of effective interventions, which are consistent with professional societies' recommendations.

\section{Acknowledgements}

The authors thank Marisa Conte for her help with developing the search algorithms for the review.

Disclosures: Drs. Auger and Kenyon received salary support from the Robert Wood Johnson Foundation Clinical Scholars program. Dr. Feudtner does not have any funding sources to disclose. Dr. Davis is funded in part by the Michigan Department of Community Health to serve as the Chief Medical Executive. The views expressed herein are not necessarily the views of the Department of Community Health. The authors have no conflicts of interest to report.

\section{References}

1. Co JPT, Ferris TG, Marino BL, Homer CJ, Perrin JM. Are hospital characteristics associated with parental views of pediatric inpatient care quality? Pediatrics. 2003;111(2):308-314. 
2. Kripalani S, LeFevre F, Phillips CO, Williams MV, Basaviah P, Baker DW. Deficits in communication and information transfer between hospital-based and primary care physicians: implications for patient safety and continuity of care. JAMA. 2007;297(8):831-841.

3. Harlan GA, Nkoy FL, Srivastava R, et al. Improving transitions of care at hospital discharge-implications for pediatric hospitalists and primary care providers. J Healthc Oual. 2010;32(5):51-60.

4. Freed GL, Uren RL. Hospitalists in children's hospitals: what we know now and what we need to know. J Pediatr. 2006;148(3):296299.

5. Forster AJ, Murff HJ, Peterson JF, Gandhi TK, Bates DW. The incidence and severity of adverse events affecting patients after discharge from the hospital. Ann Intern Med. 2003;138(3):161-167.

6. Forster AJ, Clark HD, Menard A, et al. Adverse events among medical patients after discharge from hospital. CMAJ. 2004;170(3):345-349.

7. Moore C, Wisnivesky J, Williams S, McGinn T. Medical errors related to discontinuity of care from an inpatient to an outpatient setting. $J$ Gen Intern Med. 2003;18(8):646-651.

8. Berry JG, Toomey SL, Zaslavsky AM, et al. Pediatric readmission prevalence and variability across hospitals. JAMA. 2013;309:372380 .

9. Shepperd S, Lannin NA, Clemson LM, McCluskey A, Cameron ID, Barras SL. Discharge planning from hospital to home. Cochrane Database Syst Rev. 2013;(1):CD000313.

10. Hain PD, Gay JC, Berutti TW, Whitney GM, Wang W, Saville BR. Preventability of early readmissions at a children's hospital. Pediatrics. 2012;131(1):e171-e181.

11. Feudtner C, Pati S, Goodman DM, et al. State-level child health system performance and the likelihood of readmission to children's hospitals. J Pediatr. 2010;157(1):98-102.e1.

12. Ohio Children's Hospitals' solutions for patient safety. Available at: http://solutionsforpatientsafety.org/files/sps-fact-sheet.pdf. Accessed July 24, 2013.

13. American Academy of Pediatrics. Value in inpatient pediatrics (VIP) network projects. Available at: http://www.aap.org/en-us/professionalresources/practice-support/quality-improvement/Quality-ImprovementInnovation-Networks/Pages/Value-in-Inpatient-Pediatrics-NetworkProjects.aspx. Accessed July 24, 2013.

14. Child Health Corporation of America. Resources for managing the patient discharge process. Available at: http:/www.chca.com/news/ index.html. Accessed October 31, 2013.

15. The World Bank. World Development Indicators 2012. Available at: http://data.worldbank.org/sites/default/files/wdi-2012-ebook.pdf. Accessed July 5, 2013.

16. The Cochrane Collaboration. Data collection form: Intervention review-RCTs and non-RCTs. Available at: http://hiv.cochrane.org/ sites/hiv.cochrane.org/files/uploads/Data-

20extraction\%20form_all\%20studies.docx. Accessed July 24, 2013.

17. Downs SH, Black N. The feasibility of creating a checklist for the assessment of the methodological quality both of randomised and non-randomised studies of health care interventions. J Epidemiol Community Health. 1998;52(6):377-384.

18. Aboutanos MB, Jordan A, Cohen R, et al. Brief violence interventions with community case management services are effective for high-risk trauma patients. J Trauma. 2011;71(1):228-237.

19. Shibru D, Zahnd E, Becker M, Bekaert N, Calhoun D, Victorino GP. Benefits of a hospital-based peer intervention program for violently injured youth. I Am Coll Surg. 2007;205(5):684-689.

20. Becker MG, Hall JS, Ursic CM, Jain S, Calhoun D. Caught in the crossfire: the effects of a peer-based intervention program for violently injured youth. I Adolesc Health. 2004;34(3):177-183.

21. Davis AM, Benson M, Cooney D, Spruell B, Orelian J. A matchedcohort evaluation of a bedside asthma intervention for patients hospitalized at a large urban children's hospital. I Urban Health. 2011; 88(suppl 1):49-60.

22. Espinoza-Palma T, Zamorano A, Arancibia F, et al. Effectiveness of asthma education with and without a self-management plan in hospitalized children. J Asthma. 2009;46(9):906-910.

23. Ng DKK, Chow P-Y, Lai W-P, Chan K-C, And B-LT, So H-Y. Effect of a structured asthma education program on hospitalized asthmatic children: a randomized controlled study. Pediatr Int. 2006;48(2):158162.

24. Stevens CA, Wesseldine LJ, Couriel JM, Dyer AJ, Osman LM, Silverman M. Parental education and guided self-management of asthma and wheezing in the pre-school child: a randomised controlled trial. Thorax. 2002;57(1):39-44.

25. Wesseldine LJ, McCarthy P, Silverman M. Structured discharge procedure for children admitted to hospital with acute asthma: a randomised controlled trial of nursing practice. Arch Dis Child. 1999;80(2): 110-114.
26. Madge P, McColl J, Paton J. Impact of a nurse-led home management training programme in children admitted to hospital with acute asthma: a randomised controlled study. Thorax. 1997;52(3):223-228.

27. Taggart VS, Zuckerman AE, Sly RM, et al. You Can Control Asthma: evaluation of an asthma education program for hospitalized inner-city children. Patient Educ Couns. 1991;17(1):35-47.

28. Mitchell EA, Ferguson V, Norwood M. Asthma education by community child health nurses. Arch Dis Child. 1986;61(12):1184-1189.

29. Caliskan Yilmaz M, Ozsoy SA. Effectiveness of a discharge-planning program and home visits for meeting the physical care needs of children with cancer. Support Care Cancer. 2009;18(2):243-253.

30. Broyles RS, Tyson JE, Heyne ET, et al. Comprehensive follow-up care and life-threatening illnesses among high-risk infants: a randomized controlled trial. JAMA. 2000;284(16):2070-2076.

31. Finello KM, Litton KM, deLemos R, Chan LS. Very low birth weight infants and their families during the first year of life: comparisons of medical outcomes based on after care services. J Perinatol. 1998; $18(5): 365-371$

32. Kotagal UR, Perlstein PH, Gamblian V, Donovan EF, Atherton HD. Description and evaluation of a program for the early discharge of infants from a neonatal intensive care unit. I Pediatr. 1995;127(2): 285-290.

33. Casiro OG, McKenzie ME, McFadyen L, et al. Earlier discharge with community-based intervention for low birth weight infants: a randomized trial. Pediatrics. 1993;92(1):128-134.

34. Brooten D, Kumar S, Brown LP, et al. A randomized clinical trial of early hospital discharge and home follow-up of very-low-birth-weight infants. N Engl J Med. 1986;315(15):934-939.

35. Cibulskis CC, Giardino AP, Moyer VA. Care transitions from inpatient to outpatient settings: ongoing challenges and emerging best practices. Hosp Pract (1995). 2011;39(3):128-139.

36. Hansen LO, Young RS, Hinami K, Leung A, Williams MV. Interventions to reduce 30-day rehospitalization: a systematic review. Ann Intern Med. 2011;155(8):520-528.

37. Lye PS. Clinical report-physicians' roles in coordinating care of hospitalized children. Pediatrics. 2010;126(4):829-832.

38. Greiner A. White space or black hole: what can we do to improve care transitions? ABIM Foundation. Available at: http://www.abimfoundation.org/ /media/Files/Publications/F06-05-2007_6.ashx. Accessed September 5, 2012.

39. Ivers N, Jamtvedt G, Flottorp S, et al. Audit and feedback: effects on professional practice and healthcare outcomes. Cochrane Database Syst Rev. 2012;(6):CD000259.

40. Winickoff JP, Hillis VJ, Palfrey JS, Perrin JM, Rigotti NA. A smoking cessation intervention for parents of children who are hospitalized for respiratory illness: the stop tobacco outreach program. Pediatrics. 2003;111(1):140-145.

41. Ralston S, Roohi M. A randomized, controlled trial of smoking cessation counseling provided during child hospitalization for respiratory illness. Pediatr Pulmonol. 2008;43(6):561-566.

42. Resnicow K, Page SE. Embracing chaos and complexity: a quantum change for public health. Am J Public Health. 2008;98(8):1382-1389.

43. Leschke J, Panepinto JA, Nimmer M, Hoffmann RG, Yan K, Brousseau DC. Outpatient follow-up and rehospitalizations for sickle cell disease patients. Pediatr Blood Cancer. 2012;58(3):406-409.

44. Gill JM, Mainous AG III, Nsereko M. Does having an outpatient visit after hospital discharge reduce the likelihood of readmission? Del Med J. 2003;75(8):291-298.

45. Schatz M, Rachelefsky G, Krishnan JA. Follow-up after acute asthma episodes. Proc Am Thorac Soc. 2009;6(4):386-393.

46. Berry JG, Hall DE, Kuo DZ, et al. Hospital utilization and characteristics of patients experiencing recurrent readmissions within children's hospitals. JAMA. 2011;305(7):682-690.

47. Fisher EB, Strunk RC, Highstein GR, et al. A randomized controlled evaluation of the effect of community health workers on hospitalization for asthma: the asthma coach. Arch Pediatr Adolesc Med. 2009; 163(3):225-232.

48. Krieger JW, Takaro TK, Song L, Weaver M. The Seattle-King County Healthy Homes Project: a randomized, controlled trial of a community health worker intervention to decrease exposure to indoor asthma triggers. Am I Public Health. 2005;95(4):652-659.

49. Coleman EA, Smith JD, Frank JC, Eilertsen TB, Thiare JN, Kramer AM. Development and testing of a measure designed to assess the quality of care transitions. Int J Integr Care. 2002;2:e02.

50. Parry C, Mahoney E, Chalmers SA, Coleman EA. Assessing the quality of transitional care: further applications of the care transitions measure. Med Care. 2008;46(3):317-322.

51. Berry JG, Ziniel SI, Freeman L, et al. Hospital readmission and parent perceptions of their child's hospital discharge. Int I Oual Health Care. 2013;25(5):573-581.

52. Weiss ME, Piacentine LB. Psychometric properties of the Readiness for Hospital Discharge Scale. J Nurs Meas. 2006;14(3):163-180. 